\title{
Causas e Conseqüências Físicas e Emocionais do Término de Carreira Esportiva
}

\section{Physical and Emotional Causes and Consequences of Career Termination in Sports}

Marisa Cury Agresta',

Maria Regina Ferreira Brandão²

Turíbio Leite de Barros Neto ${ }^{1}$

1. Cemafe - Centro de Medicina da Atividade Física e do Esporte. Universidade Federal de São Paulo - Departamento de Reabilitação, São Paulo, SP.

2. Universidade São Judas Tadeu, São Paulo, SP.

Endereço para correspondência: Marisa Cury Agresta. Av. Jandira, 295 - cj. 710 - 04080-001 - São

Paulo, SP

E-mail:

agrestamarisa@yahoo.com.br

Baseado na dissertação de mestrado apresentada ao Programa de Pós-graduação em Reabilitação - Escola Paulista de Medicina Universidade Federal de São Paulo, em 2006.

Submetido em 22/08/2006 Versão final recebida em 23/03/2008 Aceito em 05/07/2008

\begin{abstract}
RESUMO
Introdução: Os atletas de alto rendimento, em determinado momento, se defrontam com o término de carreira esportiva, um processo crucial e inevitável, com exigências de ajustamentos nas esferas da vida ocupacional, financeira, social e psicológica e, que, portanto, pode ser acompanhado por distress emocional. Objetivo: O presente estudo teve como objetivo pesquisar como ex-atletas brasileiros de alto nível de basquetebol e futebol profissional experienciaram a aposentadoria da carreira esportiva, suas causas e conseqüências físicas e emocionais. Métodos: Para tanto, foram avaliados 79 ex-atletas de alto nível, do sexo masculino, que pertenciam a duas modalidades esportivas: futebol $(N=57)$ e basquetebol $(N=22)$, com média de idade de $51,75 \pm 8,85$ anos. O tempo de prática como atleta profissional foi de 18,22 $\pm 4,66$ anos e o término da carreira esportiva ocorreu em média aos 34,36 $\pm 4,42$ anos. Utilizou-se uma entrevista semi-estruturada; os dados foram analisados pela freqüência de ocorrência de respostas para cada item da entrevista. Resultados: Observou-se que, para $75,9 \%$ dos atletas, a decisão de encerrar a carreira foi espontânea. A idade $(49,4 \%)$ e outros interesses emergentes $(43,0 \%)$ foram as principais causas da aposentadoria. Os sentimentos vivenciados nesse momento foram de tristeza $(50,6 \%)$ e de conformismo (36,7\%). Para 43\% dos ex-atletas a condição física piorou após o término da carreira. Conclusões: Os autores concluem que, apesar de os atletas terem experienciado uma carreira esportiva longa, o momento de se aposentar no esporte trouxe sentimentos de tristeza. Por outro lado, a idade é um limitador para a carreira esportiva e reconhecer isso levou ao conformismo.
\end{abstract}

Palavras-chave: aposentadoria, esportes/psicologia, futebol, basquetebol.

\begin{abstract}
Introduction: Elite athletes will have to face sport career termination sooner or later, and this is a crucial and inevitable process that brings about changes in the occupational, financial, social and psychological spheres of their lives, which can be followed by emotional distress. Objective: The present study had the aim to research how former Brazilian basketball and professional soccer players have experienced retirement in sports career as well as its causes and consequences. Material and Methods: 79 former elite male athletes (soccer and basketball players) with mean age of $51.75 \pm 8.85$ years old were assessed. They have been professional athletes for $18.22 \pm 4.66$ years and had their career termination at about $34.36 \pm 4.42$ years. A semi-structured interview frame was used and the data were analyzed according to the answers frequency of occurrence for each interview item. Results: It was observed that for $75.9 \%$ of these athletes the retirement from sport was their own choice. Age (49.4\%) and appearance of other interests (43.0\%) were the most frequent reasons related to the retirement. However, they have experienced feelings of sadness (50.6\%) and resignation (36.7\%) and $43 \%$ of the former athletes have experienced worsening in their physical condition after career termination. Conclusion: We can conclude that the hereby-assessed athletes have experienced a long sports career, but their retirement caused feelings of sadness. On the other hand, old age itself is a limitation for the practice of elite sports, and its acknowledgement has led to resignation.
\end{abstract}

Keywords: retirement, sports/ psychology, soccer, basketball.

\section{INTRODUÇÃO}

A Psicologia do Esporte contemporânea está em um momento de grande expansão no seu campo de conhecimentos. Acompanhando essa expansão estão surgindo novas possibilidades de pesquisas e interesses em áreas até então pouco exploradas. Dentro desse contexto, o tópico término de carreira esportiva tornou-se uma tendência mundial de investigação, uma vez que se refere a um momento crucial e inevitável na vida de um desportista, e requer, portanto, um cuidado especial. Orlick ${ }^{(1)}$ comenta a esse respeito que "(...) um tipo particular de transição que tem o potencial de interessar aos profissionais da área do esporte para investigações é a aposentadoria no alto nível, já que essa fase é inevitável" (p.138). Além do mais, a carreira esportiva, quando comparada com outras, pode ser considerada relativamente curta, exigindo um estudo mais específico e particular. Dessa forma, estudar o término da carreira esportiva, suas causas e conseqüências, faz o tema desta pesquisa ser relevante, pois permite avaliar o impacto desse momento, que requer grandes ajustamentos no estilo de vida dos atletas. 
Para tornar-se um atleta de elite, no mundo moderno, é necessário ter disciplina para treinar por muitos anos, dedicação quase que exclusiva para o esporte e, em geral, iniciar a carreira esportiva em idades muito precoces. Brandão(2) afirma que é grande a expectativa dos jogadores de alto nível de se tornarem atletas de sucesso nacional e internacional, já que essa conquista mobiliza a atenção de investimentos financeiros e tem grande espaço na mídia. Nesse contexto, os atletas profissionais ganham, como conseqüência, vantagens econômicas, notoriedade e, de fato, prestígio. Para os atletas de alto rendimento, o esporte é a energia que move a vida, é o marco de sua identidade (Brandão et al.) $)^{(3)}$. Entretanto, após anos de dedicação, por razões diversas, defrontam-se com o processo final de carreira do esporte. É quando se conscientizam de não terem sido preparados para enfrentar uma vida pós-esporte, pois negligenciaram a necessidade de um preparo para esse momento, iludidos de que a fama iria durar para sempre. Dessa forma, a aposentadoria pode gerar uma situação de estresse e de crise de identidade para muitos atletas, como podemos verificar na citação de uma ex-atleta do remo: "Eu perdi minha identidade como pessoa" (Winterstein et al.) ${ }^{(4)}$.

Para a Federação Européia de Psicologia do Esporte e Atividades Corporais (FEPSAC) ${ }^{(5)}$, identidade esportiva deve ser conceituada como a força e a exclusividade em que o esportista identifica a si mesmo com o esporte; essa identificação exclusiva é fortalecida pelo reconhecimento social e sucesso econômico que acompanham os resultados positivos da carreira esportiva. Sendo assim, terminar a carreira esportiva pode se tornar um dos momentos mais difíceis da vida de um atleta, já que a mudança no estilo de vida requer uma adaptação de papéis sociais e profissionais (FEPSAC) ${ }^{(5)}$. A maioria dos atletas não percebe a importância de outras fontes de identificação em outras esferas da vida (outra profissão ou atividade), indispensáveis para a manutenção do equilíbrio pessoal durante e após o final da carreira e isso pode ser reforçado por técnicos, dirigentes e membros da família, pois estão mais "interessados" nos resultados (conquistados por horas de exclusiva dedicação), do que em seu crescimento pessoal e profissional. A análise de Rubio(6) também confirma que, "na relação entre o ego e o desempenho de papéis sociais, muitas vezes o atleta se vê identificado com a figura espetacular sugerida pela condição de esportista, aquele capaz de realizar grandes feitos, dificultando sua participação em situações da vida cotidiana e em outras atividades sociais" (p. 97).

De acordo com Alfermann ${ }^{(7)}$, a carreira esportiva é composta de uma seqüência de sucessivas fases, com períodos de transição, identificadas como: a transição do esporte infantil para o juvenil, seguida da transição para o júnior e, finalmente, para o adulto; a transição do esporte amador para o profissional e a transição para o término da carreira esportiva. A transição de carreira esportiva significa mudança de uma fase da carreira para outra, acompanhada por concomitantes mudanças nas características psicológicas e sociais do atleta, e da necessidade de recursos para lidar com o momento. Cada uma requer exigências específicas e ajustamentos nas esferas da vida ocupacional, financeira, social e psicológica do atleta e, fundamentalmente, sempre será necessário o esforço pessoal para a adaptação à nova fase. Algumas transições para o término de carreira podem ser positivas, quando existem precondições para o ajustamento. Mas, para Stambulova ${ }^{(8)}$, nem sempre acontecem com sucesso e podem ser vivenciadas de forma negativa, quando há falta de habilidade de ajustamento, gerando sintomas psicológicos, como tristeza e depressão.
Para Crook \& Robertson ${ }^{(9)}$ e Wylleman et al. ${ }^{(10)}$, a natureza do ajustamento à aposentadoria, para cada atleta dependerá da interação de múltiplos fatores. Entretanto, um fator isolado não garante se um ajustamento será fácil e tranqüilo. Somente uma análise da complexa interação entre diversos fatores é que levará os pesquisadores a compreensão de como será sua adaptação à aposentadoria.

Dessa forma, o término da carreira esportiva é o resultado de muitos fatores e, freqüentemente, uma combinação de fatores individuais e influências sociais. Apresenta como causas uma variedade de razões, que podem ser voluntárias (decisão por livre escolha) ou involuntárias (sem livre escolha). Como exemplo, podemos citar a idade, novos interesses emergentes, fadiga psicológica, dificuldades com a equipe técnica, resultados esportivos em declínio, problemas de contusão ou de saúde, e o não-selecionamento para os jogos (Wylleman et al.) $)^{(11)}$.

Partindo do pressuposto de que a transição de carreira esportiva deve ser analisada como um processo, Taylor \& Ogilvie ${ }^{(12)}$ desenvolveram o Modelo Conceitual da Transição de Carreira, que integre, além da informação teórica da Psicologia do Esporte, a investigação empírica. As características da transição incluem: duração, mudanças de posição social, grau de estresse, desafios enfrentados e, fundamentalmente, a percepção de estresse nesse momento.

Sinclair \& Orlick ${ }^{(13)}$ também sugerem que toda transição de carreira tem o potencial de ser uma crise, alívio, ou uma combinação de ambos, dependendo da avaliação dos atletas frente à situação.

Determinadas áreas específicas, consideradas moduladoras do ajustamento ao momento da aposentadoria e facilitadoras de adaptação adequada, também podem ser avaliadas: percepção de controle; identidade esportiva; suporte social; experiências anteriores (outras transições); envolvimento com atividades relacionadas com esporte depois da aposentadoria; grau de planejamento profissional; status socioeconômico; habilidades (persistência, competitividade, metas, etc.); objetivos relacionados com esportes; e, finalmente, foco depois da aposentadoria (Lavallee ${ }^{(14)}$.

O objetivo do presente estudo foi estudar as causas e conseqüências físicas e emocionais da aposentadoria no esporte.

\section{MÉTODOS}

Para fins da presente pesquisa, foi utilizada a metodologia quantitativa e o desenho metodológico foi o estudo exploratório retrospectivo. Optou-se por esse modelo metodológico, por estar baseado em pesquisas delineadas para a investigação científica da aposentadoria, utilizando perguntas, em forma de entrevistas ou questionários, para coletar informações sobre as causas do término de carreira e caracterizar os fatores que mediam a qualidade dessa transição (Wylleman et al. $\left.{ }^{(10)}\right)$.

Participaram da pesquisa 79 ex-atletas de alto nível, do sexo masculino, de nacionalidade brasileira, que competiram em eventos de grande porte de nível nacional e internacional. Os sujeitos avaliados pertenciam a duas modalidades esportivas: futebol profissional ( $N=$ 57) e basquetebol $(\mathrm{N}=22)$.

A média de idade do grupo foi de 51,75 \pm 8,85 anos. O tempo de prática como atleta profissional foi de 18,22 \pm 4,66 anos e o término da carreira esportiva ocorreu em média aos 34,36 \pm 4,42 anos.

0 instrumento utilizado foi uma entrevista semi-estruturada com perguntas baseadas em dois questionários amplamente utilizados na literatura internacional, desenvolvidos para o estudo da Transição de Carreira Esportiva e da Aposentadoria, o APAQ - Athletic and Postath- 
letic Questionnaire (Hackfort et al.)(15) e o Sport Career Transitions (Stambulova) ${ }^{(16)}$ e que abordam temas como: biografia do atleta; aspectos psicológicos e sociológicos da carreira de alto nível; causas, problemas e dificuldades com o término de carreira; emoções vivenciadas nesse processo e a condição física depois que parou de competir profissionalmente.

Os ex-atletas foram avaliados no Ginásio de Esportes de São Paulo, no Esporte Clube Sírio, e durante o Campeonato de Veteranos de Basquetebol (realizado em Santos, julho de 2005). As entrevistas foram aplicadas individualmente, uma única vez, com duração média da 60 minutos, gravadas e posteriormente transcritas.

\section{Método estatístico}

Os dados foram analisados pela freqüência de ocorrência de respostas para cada item da entrevista.

Além do mais, com o objetivo de compreender o contexto aposentadoria e a dinâmica dos fenômenos psicológicos desse momento, segundo a percepção dos próprios jogadores, foram utilizados "indicadores de vivência" que, de acordo com Brandão(17) permitem descrever o status dos fatos em função da experiência dos sujeitos. Assim, foram utilizados depoimentos próprios dos jogadores de futebol e basquetebol, obtidos durante a aplicação da entrevista e depoimentos publicados na imprensa escrita.

\section{Procedimentos éticos}

O protocolo deste estudo foi aprovado pelo Comitê de Ética em Pesquisa da Universidade Federal de São Paulo/Escola Paulista de Medicina, CEP no 1233/05.

Os entrevistados receberam um termo de consentimento livre esclarecido, ficando claro que seus nomes não seriam divulgados e que as entrevistas seriam gravadas, diante de sua permissão. Também foram informados de que os pesquisadores se comprometiam a utilizar as informações gravadas somente para este estudo.

\section{RESULTADOS}

Os dados mostram que para 75,9\% dos ex-jogadores avaliados, a decisão de encerrar a carreira esportiva foi espontânea, ou seja, foi feita de forma voluntária. A transição de carreira ocorreu de forma gradativa e natural, como eles mesmos citaram nas entrevistas. Para a maioria dos ex-atletas (68,4\%), esse momento foi oportuno, ou seja, aconteceu na época certa, enquanto que, para 27,9\% dos entrevistados, a decisão de parar de competir profissionalmente ocorreu muito cedo e de forma prematura.

Neste estudo, as causas mais freqüentes relacionadas à saída do esporte em ordem decrescente foram: idade (49,4\%), surgimento de outros interesses (43,0\%), mudanças no estilo de vida (17,7\%), problemas de saúde (16,5\%), problemas de lesões (15,2\%), ausência de perspectivas futuras (13,9\%), problemas de relacionamento com dirigentes (13,9\%), "declínio dos resultados" (12,7\%), cansaço psicológico (11,4\%), relacionamento com o técnico (10,1\%), cansaço físico $(7,6 \%)$, relacionamento com a família $(7,6 \%)$ e relacionamento com a equipe $(3,8 \%)$.

Com relação às conseqüências emocionais vivenciadas no processo de final de carreira esportiva, observamos que algumas emoções preponderaram. Em ordem decrescente de escolhas, observamos que $50,6 \%$ dos ex-jogadores sentiram tristeza no momento da transição esportiva para uma nova carreira e 36,7 \% se sentiram conformados. Verificamos que $17,7 \%$ da amostra sentiram felicidade nesse momento, 6,3\% se sentiram tensos, 5,1\% sentiram medo,
5,1\% ficaram deprimidos, 3,8\% sentiram raiva, 3,8\% ficaram ressentidos, 1,3\% sentiram culpa e nenhum dos entrevistados fez referência a sentimentos de desespero.

Com relação às conseqüências físicas de encerrar a carreira esportiva, os dados mostram a condição física atual dos ex-atletas avaliados e indicam que 43\% dos entrevistados tiveram a percepção de que a aptidão física piorou depois de terem se aposentado. Segundo dados coletados nas entrevistas, o peso dos sujeitos aumentou e estão "quase" sedentários, ou seja, sua rotina se resume a praticar apenas "alguns exercícios físicos", como uma caminhada. Um dado importante é que a condição física não melhorou para nenhum dos avaliados.

\section{DISCUSSÃO}

Embora o Brasil tenha conquistado o título de pentacampeão mundial de futebol e de bicampeão mundial de basquetebol, a literatura nacional é escassa sobre esse tema. É surpreendente notar que não existe a preocupação para se entender o processo pelo qual passam os atletas em transição para uma vida pós-carreira esportiva, momento que talvez represente uma das passagens mais importantes em sua vida, e o impacto disso em todas as esferas.

Os dias de brilhantismo e prestígio, tão desejados no início de uma carreira esportiva, chegam ao final, já que a aposentadoria é inevitável. A transição de uma vida esportiva para uma vida pós-esporte não se dá de forma fácil; muitas vezes, não existe suporte dos dirigentes esportivos e os jogadores, em geral, têm poucas possibilidades de uma nova profissão que não esteja relacionada ao ambiente esportivo (Brandão et al.) $)^{(3)}$.

Alguns estudos comentam a dor emocional e a saudade vivenciada por atletas em final de carreira, que podem se tornar ansiosos e depressivos, abusar de álcool e drogas(4-18). O distress se estabelece quando consideram o que irão fazer, uma vez que não podem mais participar do esporte como jogadores de alto nível, e os amigos, companheiros de time, jornalistas e torcedores desaparecem.

Para Brandão(17), distress indica uma reação negativa frente a estímulos do ambiente, com a percepção de uma ameaça a uma demanda imposta por uma situação particular. Nesse sentido, os atletas deste estudo vivenciaram distress no momento de transição para a aposentadoria, pois também sentiram a tristeza e a saudade de não participar mais da arena esportiva.

A citação de alguns ex-atletas ilustra essa situação. Em uma entrevista ao Jornal da Tarde, o ex-jogador Pelé comentou: "Aceitar que chegou a hora de parar é terrivel, a depressão surgiu quando a hora chegou" (Rimoli) ${ }^{(19)}$. Na mesma entrevista, o ex-jogador de basquete Oscar (43 anos) disse: "Sei que a hora de parar está chegando, ou já passou, sei lá. Só que são mais de 25 anos fazendo a mesma coisa. Não vou negar que é duro pensar que não estarei jogando mais uma partida oficial. Ainda mais porque estou bem fisicamente e não estou atrapalhando o time". A frase de um ex-atleta confirma também como é difícil encerrar a carreira profissional no esporte: "Fica eternamente a saudade" (ex-jogador de basquete, 50 anos).

Quanto ao término da carreira, a decisão para esse momento pode ser forçada ou espontânea. Para Mendelsohn ${ }^{(18)}$, na maioria das vezes, não é o jogador que se aposenta por conta própria e põe fim a sua carreira, mas é aposentado ou retirado do esporte de alguma maneira; em alguns casos, de forma paulatina, em outros, de maneira abrupta. Já para Roffé(20), existem duas situações distintas que delineiam a aposentadoria dos atletas: primeiro, quando ele se apo- 
senta voluntariamente, dentre outras razões, porque percebe que seu rendimento já está em declínio, sofreu uma lesão grave ou quando considera que já cumpriu seus objetivos, julgando que está psicologicamente cansado e o ambiente do esporte se "desgastou". Segundo, quando "o esporte" aposenta o atleta, porque não rende mais, os dirigentes e técnicos "decidem" que não está mais em condições de jogar pelo clube ou pela seleção. Quando isso ocorre, pode sofrer e se decepcionar, levando-o à aposentadoria no esporte. A frase a seguir exemplifica isto: "Me senti humilhado, o presidente do clube exigiu que eu saísse, tive problemas de relacionamento com os dirigentes" (ex-jogador de futebol, 81 anos).

Os dados do presente estudo são semelhantes aos achados de Blinde \& Greendorfer ${ }^{(21)}$, Vasconcelos ${ }^{(22)}$ e Werthner \& Orlick ${ }^{(23)}$, pois também observaram que quase a totalidade dos atletas avaliados se aposentou voluntariamente e o processo aconteceu de forma gradativa.

Podemos verificar que a causa mais freqüente relacionada à aposentadoria da amostra de atletas avaliados foi a idade avançada para a prática do esporte de alto nível, associada ao surgimento de outros interesses e motivação para mudanças no estilo de vida. A citação de ex-atletas exemplifica o motivo da idade como facilitadora para o término da carreira de alto nível:

"Senti-me aliviado, pois percebi que chegou a hora" (ex-jogador de basquete, 44 anos).

"Não dava mais" (ex-jogador de futebol, 46 anos, quando se referiu à "idade avançada" para o esporte de alto nível como a maior causa para a aposentadoria).

"Parei com 42 anos, o meu corpo não conseguia mais acompanhar as jogadas" (ex-jogador de futebol, 47 anos).

"Fui até o limite" (Brandão et al.) (3).

O processo de transição do esporte para uma nova carreira teve, como conseqüências, o sentimento de tristeza dos atletas avaliados, mas, também, de conformismo, pois perceberam que a idade os impedia de continuar e, ainda, desejavam realizar uma mudança no estilo de vida.

Segundo o atleta de basebol Nolan Ryan, a principal preocupação no momento de sua aposentadoria era lembrar a seus colegas que iriam se aposentar, de que não importaria o quanto eles planejassem esse momento, mas que estivessem prontos para um afastamento dolorido e com angústia. O ex-atleta comentou nessa entrevista que, embora tivesse se preparado financeiramente para a aposentadoria, conseguindo concretizar dois grandes sonhos - viajar com a família e dirigir uma fazenda - ainda sentia falta da competição e da sensação de ser membro de uma equipe, um sentimento que não existe fora do esporte. A transição para o término de sua carreira levou dois anos, vivenciados com distress (Brown) ${ }^{(24)}$.

Com relação aos atletas da presente pesquisa, a declaração de Pelé também mostra sentimentos de tristeza e um momento de dilemas existenciais e crise de identidade durante o processo para a aposentadoria. Porém, a motivação para outros interesses o incentivou a tomar essa decisão na época adequada:

"Tive três despedidas na minha carreira, uma na Seleção Brasileira, uma no Santos e a última no Cosmos, foi uma mais triste do que a outra" (Rimoli) $^{(19)}$.

A citação de Nunes (48 anos), ex-atacante, campeão do mundo em 1981, mostra as dificuldades de adaptação e a falta do apoio psicológico no término da carreira; só conseguiu diminuir a angústia que o perseguia desde que encerrou a carreira, em 1992, porque estava correndo e chutando bolas na Gávea (RJ): "A vida fica muito triste depois que tudo passa, sinto falta de ser reconhecido, de dar autógrafos, de viajar o mundo com os times. Até da concentração tenho saudade" (Rimoli) $^{(19)}$.

Ao abandonar o papel de atleta, com o término da carreira, é como se perdessem uma parte importante de si mesmos. A perda do status físico e social (que acompanhava os resultados obtidos nas vitórias e no reconhecimento do clube e torcedores) faz com que, agora longe das quadras ou dos campos, se sintam esquecidos e frustrados, pois não mais pertencem à arena esportiva. Essa questão é tão significativa que, para Brewer et al. ${ }^{(25)}$, o "tendão de Aquiles" dos atletas está relacionado à perda da valorização pessoal, ou seja, à "crise de identidade" no momento da aposentadoria.

Quando o atleta se mostra forte e exclusivamente identificado com a figura de esportista, pode apresentar dificuldades psicológicas no momento da aposentadoria. E esses resultados estão de acordo com os obtidos nos estudos de Werthner \& Orlick ${ }^{(23)}$, Pearson \& Petitpas ${ }^{(26)}$. A forte identidade atlética apresenta importante impacto na natureza da transição de carreira para adaptação a um novo estilo de vida, ou seja, atletas fortemente identificados com a figura de esportista podem vivenciar o momento do término de carreira com uma mudança de identidade. Nesse caso, ocorre a perda da valorização pessoal e aparecem dificuldades de adaptação para o desempenho de outros papéis sociais não-relacionados ao esporte, já que esses não foram exercidos durante a carreira no esporte.

Observou-se que a condição física da maioria dos atletas avaliados piorou depois da aposentadoria. Como conseqüência, o peso desses ex-atletas aumentou e a rotina de exercícios tem sido praticar caminhada. Semelhantes resultados foram encontrados em um estudo realizado com ex-jogadores de futebol de salão, que disputaram campeonatos nacionais e internacionais e haviam encerrado a carreira esportiva havia dois anos. Foi observado que $80 \%$ deles apresentaram piora na sua condição física após a saída do esporte (Agresta et al.) (27).

Da mesma forma, Baillie \& Danish ${ }^{(28)}$ reconhecem que é muito difícil um atleta de alto nível manter a mesma agenda de atividades físicas depois que se aposenta do esporte competitivo, ou seja, as atividades continuam, mas não na mesma intensidade.

Para Lópes ${ }^{(29)}$, a condição física atual de ex-atletas deveria ser considerada de grande importância, já que muitos apresentam aumento de peso, perda da massa muscular e problemas emocionais, como a depressão e a ansiedade, como conseqüências da aposentadoria no esporte. $\mathrm{O}$ autor acrescenta que algumas manifestações físicas e psicológicas podem ocorrer em ex-atletas como resultado da inatividade e pela falta de um programa de "destreinamento". Podem aparecer hipertensão arterial e suas complicações, dores de cabeça, isquemia e infarto, aumento do peso corporal, fadiga mental, aumento do consumo de álcool e de cigarro. Nos estudos do autor, verificou-se que jogadores que continuavam ativos fisicamente sentiam-se melhor (sensação de bem-estar e humor estável) do que jogadores sedentários.

Mello et al. ${ }^{(30)}$ têm investigado como ocorre a modulação dos estados de humor com a prática de exercícios físicos (alta intensidade ou moderada). Segundo os autores, esses estudos poderão fornecer dados sobre a intensidade e a duração adequada do exercício físico, para que sejam verificados efeitos positivos em sintomas ansiosos e depressivos.

De toda forma, a investigação de Lópes ${ }^{(29)}$ mostrou a necessidade de o jogador fazer um "destreinamento esportivo", um processo que se 
inicia a partir da aposentadoria e tem objetivo profilático para sua saúde. O programa de "destreinamento" propõe a diminuição planificada e gradativa da carga física a que foram submetidos durante o período ativo e faz o ex-atleta compreender que encerrar a carreira esportiva não significa o abandono da atividade física e do exercício.

Apesar da relevância de todos esses dados, este estudo apresenta como limitação o fato de que a amostra avaliada encerrou a carreira esportiva em média há 16,39 \pm 8,97 anos. Isso quer dizer que não foram avaliados durante o processo de transição, o que pode ter levado à perda de informações importantes sobre o sentimento vivenciado nesse momento. Entretanto, cabe uma ressalva no sentido de que, mesmo após tanto tempo, os ex-jogadores foram capazes de passar o sentimento experienciado, o que nos mostra o quão relevante é o estudo da aposentadoria no esporte.

\section{CONCLUSÃO}

A aposentadoria pode ser caracterizada por uma crise, alívio, ou uma combinação de ambos, dependendo da percepção dos atletas frente ao momento em que ela ocorre. Com isso, pode-se concluir que deixar a arena esportiva tende a ser um momento difícil da vida de um atleta, pois sempre requer adaptação de papéis sociais e profissionais. Fica claro que essa adaptação torna-se ainda mais difícil quando o atleta tem forte identificação com a figura de esportista. Em geral, apresenta dificuldades psicológicas e vivencia momentos de tristeza, depois de anos de exclusiva dedicação à carreira competitiva.

\section{REFERÊNCIAS BIBLIOGRÁFICAS}

1. Orlick T. From hero to zero. In: In pursuit of excellence: how to win in sport and life through mental training. 2nd ed. Champaign: Human Kinetics, 1990;167-75.

2. Brandão MRF. O lado mental do futebol. In: Barros Neto TL, Guerra I, editores. Ciência do futebol. Barueri: Manole, 2004;203-20.

3. Brandão MRF, Winterstein P, Agresta M, Pinheiro C, Akel MC, Martini L. Career transitions of former brazilian top level athletes. In: Papaioannou A, Goudas M, Theodorakis Y. In the dawn of the new millennium. Programme and Proceedings of the 10th World Congress of Sport Psychology; 2001 May 28-Jun 2; Skiathos, Greece. Thessaloniki: Christodoulidi, 2001;1-2.

4. Winterstein P, Brandão MRF, Pinheiro C, Agresta M, Akel CM, Martini L. Transition in sports career in brazilian professional soccer players. In: Papaioannou A, Goudas M, Theodorakis Y. In the dawn of the new millennium. Programme and Proceedings of the 10th World Congress of Sport Psychology; 2001 May 28-Jun 2; Skiathos, Greece. Thessaloniki: Christodoulidi, 2001;3-4.

5. FEPSAC. Position statement: sports career termination, 1999. Biel: FEPSAC; c2003a [cited 2006 Jan 9]. Available from: http://www.smartstep.se/ssp/sportpsychology/module.asp?page=detail\&XMod uleld $=8243 \&$ Productld $=2670$.

6. Rubio K. O atleta e o mito do herói: o imaginário esportivo contemporâneo. São Paulo: Casa do Psicólogo, 2001.

7. Alfermann D. Career transition and concomitant changes in athletes. In: Proceedings of the 11th World Congress of Sport Psychology; 2005 Aug 5-19; Sidney, Austrália. Sidney: International Society of Sport Psychology, 2005.

8. Stambulova N. Transitional period of russian athletes following sports career termination. In: Lidor R, Bar-Eli M. Proceedings of the IXth World Congress of Sport Psychology; 1997 Jul 5-9; Netanya, Israel. Israel: International Society of Sport Psychology, 1997;658-60.

9. Crook JM, Robertson SE. Transitions out of elite sport. Int J Sport Psychol 1991;22:115-27.

10. Wylleman P, Stambulova NB, Biddle S. Career transitions in sport: research and interventions. In: Proceedings of the 10th European Congress of Sport Psychology; 1999 Jul 7-12; Prague, Czech Republic. Prague: Charles University Press, 1999;301-3.

11. Wylleman P, Lavallee D, Alfermann D, editors. Career transitions in competitive sports. Biel (Switzerland): FEPSAC, 1999. [FEPSAC Monograph Series, 1].

12. Taylor J, Ogilvie BC. A conceptual model of adaptation to retirement among athletes. J Appl Sport Psychol 1994;6:1-20.

13. Sinclair DA, OrlickT. Positive transitions from high-performance sport. Sport Psychol 1993;7:138-50.

14. Lavallee D. The effect of a life development intervention on sports career transition adjustment. Sport Psychol 2005;19:193-202.
Os dados evidenciam o quanto a idade é um fator limitador da performance e desencadeador da aposentadoria, o que acaba levando a um sentimento de conformismo e, ao mesmo tempo, motivando para outros interesses e objetivos emergentes, tais como o desejo de poder passar mais tempo com a família.

Chama-nos a atenção a piora na saúde física dos ex-atletas e o quanto a diminuição planificada e gradativa da carga física a que foram submetidos durante o período ativo é necessária, ao se pensar na qualidade de vida pós-esporte.

Dessa forma, diante dos achados nesta pesquisa de que a aposentadoria no esporte pode refletir sentimentos negativos e dificuldades de ajustamento, emerge a necessidade, tanto do campo científico, como da direção esportiva, de desenvolver programas de aposentadoria que tenham como objetivo principal capacitar atletas em transição de carreira a lidarem melhor com esse momento e, principalmente, utilizar esses programas no início da carreira para minimizar a ansiedade com relação ao futuro. Nesse sentido, propomos, como sugestão, a elaboração de programas de aposentadoria, inéditos no Brasil, para que sejam empregados durante a carreira do atleta e que tenham o apoio de Federações, Confederações e do Comitê Olímpico Brasileiro, tendo em vista sua fundamental importância para uma integração positiva na vida pós-atlética, prevenindo possíveis desordens emocionais.

Todos os autores declararam não haver qualquer potencial conflito de interesses referente a este artigo.

15. Hackfort D, Emrich E, Papathanassiou V. Nachsportliche karriereverläufe. Schorndorf: Hofmann, 1997.

16. Stambulova NB. Sports career transitions of russian athletes. In: Vanbraechem-Raway R, Auweele V, editors. Proceedings of the IXth European Congress on Sport Psychology, 1995;4-9; Brussels, Belgium. Brussels: FEPSAC, 1995;867-73.

17. Brandão MRF. Fatores de stress em jogadores de futebol profissional [tese]. Campinas: Universidade Estadual de Campinas, 2000

18. Mendelsohn D. El retiro del futbolista. Efdeportes [periódico en Internet]. 1999 oct [citado 2005 dic 12];4(16):[cerca de 4 p.]. Disponible en: http://www.efdeportes. com/efd16/retiro.htm

19. Rímoli C. Ah, como é difícil dizer adeus à fama... Jornal da Tarde. 2002 set 8; Caderno B:6.

20. Roffé M. Retiro del futbolista: el drama del día después. Efdeportes [periódico en Internet]. 2000 nov [citado 2005 oct 15];5(27):[cerca de 5 p.]. Disponible en: http://www.efdeportes.com/efd27a/retiro.htm

21. Blinde EM, Greendorfer SL. A reconceptualization of the process of leaving the role of competitive athlete. Int Rev Soc Sport 1985;20:87-94.

22. Vasconcelos P. Abandono da prática desportiva e sucesso na adaptação à vida activa em nadadores portugueses de elite. Efdeportes [periódico na Internet]. 2003 mar [citado 2005 mar 17];8(58):[cerca de 17 p.]. Disponível em: http://www. efdeportes.com/efd58/aband.htm

23. Werthner P, Orlick T. Retirement experiences of successful Olympic athletes. Int J Sport Psychol 1986;17:337-63.

24. Brown B. Execs can learn from strikeout king's transition. Career J [serial on the Internet]. 2001 May 11 [cited 2006 Feb 17]. Available from: http://www.career journal.com/myc/retirement/20010511-brown.html

25. Brewer BW, Van Raalte JL, Linder DE. Athletic identity: Hercules' muscles or Achilles heel? Int J Sports Psychol 1993;24:237-54.

26. Pearson R, Petitpas A. Transition of athletes: pitfalls and prevention. J Couns Dev 1990;69:7-10.

27. Agresta MC, Brandão MRF, Rebustini F, Ribeiro FA, Barros Neto TL. Terminación de la Carrera Deportiva en Jugadores de Futsal [Abstract ]. In: Libro de Resúmenes del II Congreso Internacional de Psicología Aplicada al Deporte, 2003;12-5; Madri, Espanha. Madri: Dykinson; 2003;137.

28. Baillie PHF, Danish SJ. Understanding the career transition of athletes. Sports Psychol 1992;6:77-98.

29. Lópes RFA. Desentrenamiento: análisis y criterios actuales. Efdeportes [periódico en Internet]. 2001 jun [citado 2005 dic 20];7(37):[cerca de 4 p.]. Disponible en: http://www.efdeportes.com/efd37/ desentr.htm

30. Mello, MT, Boscolo, RA, Esteves, AM, Tufik, S. O exercício físico e os aspectos psicobiológicos. Rev Bras Med Esporte 2005;11: 2037 